\title{
A critical assessment of the metal content of the intracluster medium
}

\author{
S. Molendi ${ }^{1}$, D. Eckert ${ }^{2,1}$, S. De Grandi ${ }^{3}$, S. Ettori ${ }^{4}$, F. Gastaldello ${ }^{1,5}$, S. Ghizzardi ${ }^{1}$, G. W. Pratt ${ }^{6}$, and M. Rossetti ${ }^{7,1}$ \\ 1 INAF-IASF Milano, via E. Bassini 15, 20133 Milano, Italy \\ e-mail: silvano@iasf-milano.inaf.it \\ 2 Department of Astronomy, University of Geneva, Ch. d'Ecogia 16, 1290 Versoix, Switzerland \\ 3 INAF-Osservatorio Astronomico di Brera, via E. Bianchi 46, 23807 Merate, Italy \\ ${ }^{4}$ INAF, Osservatorio Astronomico di Bologna, via Ranzani 1, 40127 Bologna, Italy \\ 5 Department of Physics and Astronomy, University of California at Irvine, 4129 Frederick Reines Hall, Irvine, \\ CA 92697-4575, USA \\ ${ }^{6}$ Laboratoire AIM, IRFU/Service d'Astrophysique - CEA/DSM - CNRS - Université Paris Diderot, Bât. 709, CEA-Saclay, \\ 91191 Gif-sur-Yvette Cedex, France \\ 7 Dipartimento di Fisica dell’Università degli Studi di Milano, via Celoria 16, 20133 Milano, Italy
}

Received 14 September 2015 / Accepted 12 November 2015

\section{ABSTRACT}

\begin{abstract}
Aims. Our goal is to provide a robust estimate of the metal content of the intracluster medium (ICM) in massive clusters.
Methods. We made use of published abundance profiles for a sample of $\sim 60$ nearby systems. We included in our estimate uncertainties associated with the measurement process and with the almost total lack of information in cluster outskirts.

Results. We performed a first, albeit rough, census of metals and find that the mean abundance of the ICM within $r_{180}$ is very poorly constrained, $0.06 Z_{\odot} \lesssim Z \lesssim 0.26 Z_{\odot}$ and presents no disagreement with expectations. Similarly, whether and how the bulk of the metal content in clusters varies with cosmic time are very much open questions.

Conclusions. A solid estimate of abundances in cluster outskirts could be achieved by combining observations of the two experiments that will operate on board Athena, the XIFU and the WFI, provided they do not fall victim to the de-scoping process that has afflicted several space observatories over the last decade.
\end{abstract}

Key words. galaxies: clusters: intracluster medium - X-rays: galaxies: clusters

\section{Introduction}

Over the last decade and a half, analysis of spectra from the latest generation of X-ray experiments, has allowed the measure of density, temperature, pressure, and entropy of the intracluster medium (ICM) for several hundreds of systems. For a more limited number of objects, long dedicated observations have permitted detailed studies of cores and of other regions of particular interest. More recently, the coming of age of Sunyaev-Zel'dovich (SZ) experiments has allowed the construction of significantly less biased cluster samples that extend to greater cosmological distances than X-ray samples (e.g. Planck Collaboration XXIX 2014). All in all, the wealth of thermodynamic measures collected out to redshifts of $\sim 1$ has greatly improved our understanding of the physics of these systems and made clusters one of the major tools for estimating cosmological parameters.

$\mathrm{X}$-ray data can also be used to derive another quantity, one that cannot be measured with SZ experiments and that has been used to a lesser extent than others, namely metal abundance. Spectra of high statistical quality can be used to derive the abundance of several elements, $\mathrm{O}, \mathrm{Mg}, \mathrm{Si}, \mathrm{S}, \mathrm{Ar}, \mathrm{Ca}, \mathrm{Fe}$, and $\mathrm{Ni}$ (see Tamura et al. 2004; Mernier et al. 2015); however, for the majority of systems, measures are restricted to the most prominent line, i.e. the $\mathrm{Fe} \mathrm{K} \alpha$ line at $7 \mathrm{keV}$. These measures have been used by several workers (see de Plaa 2013 for a recent review) to infer several properties of the ICM. For example some have attempted to use metals as tracers of gas motions in the ICM (e.g. Ghizzardi et al. 2013; Rossetti \& Molendi 2010). A few (e.g. Tamura et al. 2011) have audaciously attempted to use the limited spectral resolution of X-ray CCDs to directly measure shifts in the lines ${ }^{1}$. However, most of the effort thus far has gone into trying to characterize the metal content of the ICM. Radial distributions of the metal abundance are available for several tens of systems while 2D distributions have been published for a more limited number of objects. Several authors have compared abundances of different elements to point out that the enrichment of the ICM requires contributions from both SNIa and SNcc (de Plaa et al. 2007; De Grandi \& Molendi 2009). There have been a few attempts to connect the abundance distribution with the cluster formation and evolution history (e.g. Fabjan et al. 2010); the amount of metals that end up in the ICM is expected to depend critically upon the interplay between star formation and AGN activity and can be used to provide constraints on feedback processes that are complementary to those based on the entropy distribution (e.g. Voit et al. 2005; Pratt et al. 2010). Some have attempted to gauge the enrichment process of the ICM (Tornatore et al. 2007; Cora et al. 2008) and to relate it to the nucleo-synthesis and ultimately the star formation processes in cluster galaxies. More specifically, attempts to relate

1 Things should change dramatically with the launch of the first X-ray micro-calorimeter, the Soft X-ray Spectrometer (SXS) on board the ASTRO-H mission in February 2016. With a spectral resolution of $6 \mathrm{eV}$, measurements of line shifts and broadenings associated with subsonic motions are well within the reach of the SXS. 
the observed Fe content of the ICM with that expected from the stellar population, have come to the conclusion that, for the most massive clusters, the former exceeds the latter by a factor of several (e.g. Loewenstein 2013). This is recognized as a problem because, unlike less massive systems where the potential well is sufficiently shallow to allow the escape of at least part of the metals injected into the ICM, for massive clusters all metals are expected to remain within the system. In this paper we provide a critical assessment of the metal content of the ICM. In Sect. 2 we briefly review the methodology and systematics involved in measuring abundances in the ICM. In Sect. 3 we perform our estimate of the metal content of the ICM, and in Sect. 4 we discuss our finding and their implications. In Sect. 5 we consider future prospects for the measure of the Fe content of the ICM. Finally, in Sect. 6 we summarize our results.

Abundances are reported relative to the solar photospheric values of Anders \& Grevesse (1989), where Fe $=4.68 \times 10^{-5}$ (by number relative to $\mathrm{H}$ ). We make this choice despite the significant evolution of solar reference systems over the last two decades, (see Lodders 2010 for a review). Indeed, these changes have led to the introduction of new references which have been superseded in a matter of years. In this framework, the choice of an old but widely adopted and recognized abundance reference is not such a bad one. To ease comparison with other systems we note that our Fe abundances can be converted into the reference systems proposed in Lodders (2003) and Asplund et al. (2009) by multiplying by 1.59 and 1.48 , respectively.

Throughout this paper the term "metal" will be used as a synonym for Fe.

\section{Measuring abundances}

Both continuum and line emission from the ICM are two-body processes. The continuum emission is proportional to the product of the number of all ions and the number of electrons, while the line emission is proportional to the number of ions of a given species times the number of electrons. Under these conditions it is easy to show that the equivalent width of a given line is proportional to the ratio of the number density of the element producing the line over the number density of hydrogen. In other words, the equivalent width of the line is proportional to the relative abundance of the element producing it (e.g. Sarazin 1988). Thus, even at the relatively modest resolution provided by current CCD detectors $(\sim 2 \%$ at $6 \mathrm{keV})$, intense and isolated lines (such as the $\mathrm{FeK} \alpha$ line) typically feature equivalent widths comparable to or in excess of the spectral resolution. Several authors (e.g. de Plaa 2013) have pointed out that the above conditions lead to direct and reliable estimates of the metal abundance in clusters. In 2009 we carried out a study to verify this expectation (De Grandi \& Molendi 2009). We performed a detailed spectral analysis of the spectra of the central regions of $26 \mathrm{cool}$ core clusters. These systems are bright and the spectra are of high statistical quality allowing precise estimates of the equivalent width of a few ion species. We identified and investigated three possible causes of systematic uncertainties: 1) the calibration of the X-ray experiment; 2) the plasma code used to fit the X-ray data, and 3) the thermal structure of the ICM. By comparing data from the three detectors on board XMM-Newton we found that systematic errors on the abundance were below $3 \%$ for $\mathrm{Si}$ and Fe. By comparing the mekal (Mewe et al. 1985, 1986; Kaastra 1992; Liedahl et al. 1995) and apec (Smith et al. 2001) plasma codes, available within the X-ray spectral fitting package XSPEC (Arnaud 1996), we found differences of $10 \%$ for $\mathrm{Si}$ and $5 \%$ for $\mathrm{Fe}^{2}$. Finally, by fitting spectra with different multi-temperature models, namely $2 \mathrm{~T}$ and $4 \mathrm{~T}$, we found that systematic differences were always below $3 \%$ for $\mathrm{Fe}$. In conclusion, focusing on $\mathrm{Fe}$, which is the element that is typically used to measure the metal abundance of the ICM, we found that systematic errors were below 5\%. Thus, we are able to confirm that, if data of sufficient statistical quality is available, robust and precise estimates of the metal abundance can be made.

\section{Metal content of the ICM}

The first measurement of the Fe line in clusters dates back to 40 yr ago (Mitchell 1976). Since that time much has been learned about the metal content of clusters. In the following section we review the relevant literature.

\subsection{The state of the art}

One of the first systematic studies of abundance profiles is a paper based on BeppoSAX data, which we wrote more than a decade ago (De Grandi \& Molendi 2001). In this work we showed for the first time that while non-cool-core (NCC) systems show relatively flat profiles, cool-core (CC) clusters feature an abundance excess in their centre. An important aspect of this measurement is that the profiles could be extended out to about $0.4 r_{180}{ }^{3}$. More recently, we performed a more extensive study based on a sample of 60 massive clusters ${ }^{4}$ observed with XMM-Newton (Leccardi et al. 2010). From Fig. 6 of that paper we found that the strength of the metal abundance excess correlates with the central entropy, in the sense that within roughly $0.1 r_{180}$, systems with lower central entropy feature a stronger central excess. In the intermediate region (i.e. between $0.1 r_{180}$ and $\left.0.2 r_{180}\right)$, the three groups of clusters we identified, namely low-entropy core systems (LEC), medium-entropy core systems (MEC), and high-entropy core (HEC) systems, all show an abundance excess with respect to the metallicity measured in the outer region. Beyond 0.2r $r_{180}$ we found no evidence of any difference in abundance between clusters belonging to the three classes described above.

Another important point is that this external region extends out to $0.4 r_{180}$. In other words, while the XMM-Newton observations have allowed us to achieve a significant improvement in our characterization of core and circum-core regions, they have not permitted us to extend our exploration of the metal abundance of outskirts beyond what was previously known. We have discussed the reasons for this failure elsewhere (Molendi 2004; Ettori \& Molendi 2011) and we will not review them here. However, we do wish to note that measures of metal abundance of clusters extend out to rather small radii. If we take as reference the mass of the ICM measured within $r_{180}$, as determined in Eckert et al. (2012; see their Fig. 6), we find that the gas mass within $0.1 r_{180}$ ranges between $2 \%$ and $5 \%$ of the total gas mass, depending on

\footnotetext{
2 This may have improved over the last few years; however, since there is no single fitting package containing updated versions of these codes, the comparison is somewhat complicated.

$3 r_{180}$ is defined as the radius within which the mean density is 180 times the critical density of the Universe. For massive clusters $r_{180} \simeq 1.6 r_{500}$, where $r_{500}$ is another reference radius often used in the literature, defined similarly to $r_{180}$.

4 In our works "massive" systems have mean temperatures in excess of $3.5 \mathrm{keV}$ (see Leccardi \& Molendi 2008b), roughly corresponding to $3 \times 10^{14} M_{\odot}$.
} 
how peaked the surface brightness profile is in the core; it climbs to $10 \%$ within $0.2 r_{180}$ and to $33 \%$ within $0.4 r_{180}$. In other words, the metal abundance of $2 / 3$ of the ICM is simply not known.

In recent years there has been one $e^{5}$ attempt to measure the metal abundance at larger radii with the Suzaku satellite on the Perseus cluster. Werner et al. (2013) measure a flat abundance profile out to $\sim 0.9 r_{180}$ with a mean value consistent with the one we determined in Leccardi et al. (2010). This is certainly a very interesting measure; however, it cannot be used to provide a robust estimate of the metal abundance in clusters at large radii for at least four good reasons: 1) the measure has only been performed on one system; 2) it does not provide a full azimuthal coverage of the cluster; the coverage actually decreases as a function of radius; 3 ) the measure has been performed assuming a single-temperature model, an assumption made in all the analyses of the outer regions, including our own (Leccardi \& Molendi 2008a; Leccardi et al. 2010), but one that cannot be tested with the current data and that could lead to systematic errors on the abundance (see Sect. 3.2 for details); 4) even under the unverified assumption of a single-temperature medium, abundance measurements in cluster outskirts are extremely challenging (e.g. Ettori \& Molendi 2011); when the continuum from the cluster is only a small percent of the total signal that is measured, an exquisite characterization of the background is required to provide reliable estimates of thermodynamic parameters. For the metal abundance, which typically requires the characterization of equivalent widths of a few hundred $\mathrm{eVs}$, at $6 \mathrm{keV}$ it is even more necessary. This is illustrated by the fact that while a few tens of temperature measurements around $r_{180}$ are available in the literature (e.g. Reiprich et al. 2013), there is only one for the abundance.

\subsection{Abundance bias in the outskirts}

In this section we provide an example to illustrate why metal abundances could be significantly overestimated in cluster outskirts. As pointed out in Sect. 3.1, spectra from outer regions are typically fit with one-temperature models; however, there is mounting evidence that the temperature structure is more complicated. Measurements of the surface brightness in outer regions indicate that inhomogeneities are certainly present on scales of several tens of kpc and beyond (Nagai \& Lau 2011; Roncarelli et al. 2013; Eckert et al. 2015), moreover, although not yet detected, they likely extend to significantly lower sizes (e.g. Gaspari et al. 2014). Since regions of different density will be kept in approximate pressure equilibrium by sound waves, the overdense regions will also be cooler and at a lower entropy than less dense regions. An important question is how metals are distributed between the different phases: Do all components share the same abundance or are some more metal-rich than others? We have shown that in relaxed (e.g. Ghizzardi et al. 2013) and intermediate systems (Rossetti \& Molendi 2010) entropy and metallicity tend to anti-correlate, i.e. regions of lower entropy are more metal-rich. If mixing and thermal equilibration processes are ineffective in homogenizing gas that is set into motion by the continuous accretion process in clusters, as suggested by some of our recent work (Eckert et al. 2014; De Grandi et al. 2015), it may well be that the lower entropy clumps have different, likely higher, metallicity than the surrounding medium.

\footnotetext{
There is also a measurement on the Virgo cluster (Simionescu et al. 2015); however, this is a low mass system with a total mass of roughly $1.4 \times 10^{14} M_{\odot}$ and we will not consider it further.
}

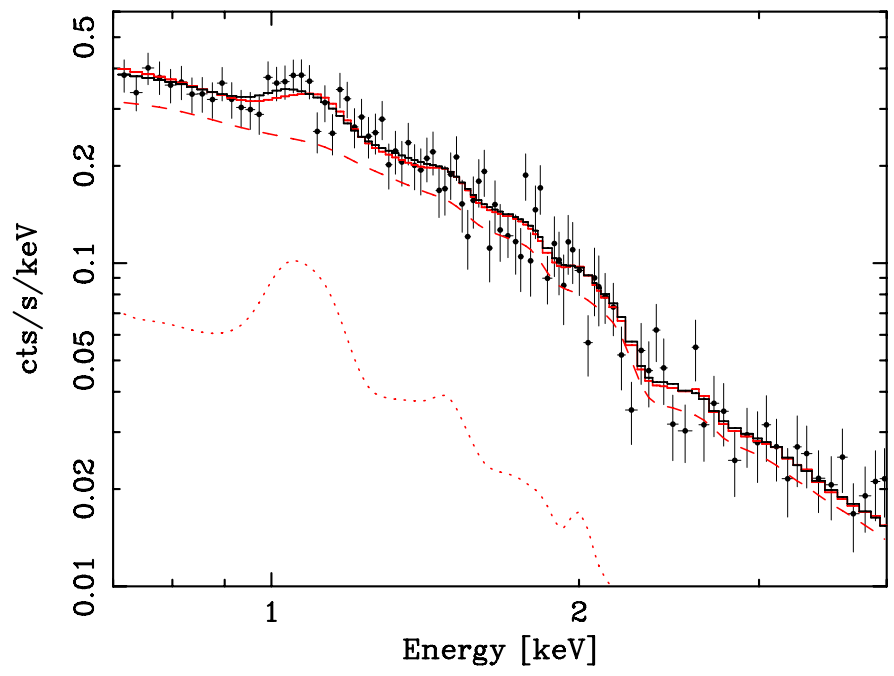

Fig. 1. Simulated two-temperature spectrum and best fitting onetemperature model. The simulated spectrum and its components are plotted in red, the $1.8 \mathrm{keV}$ and $3.6 \mathrm{keV}$ components are shown as a dotted and dashed line, respectively, while the total spectrum is shown as a solid line. The best fitting one-temperature model is shown as a solid black line. Both the simulation and the fit were performed in a broad energy band, $0.5-10.0 \mathrm{keV}$; however, here we show only the $0.7-4.0 \mathrm{keV}$ range. The one-temperature model does an adequate job of reproducing the shape of the L-shell blend arising from the two-temperature spectrum (for a more detailed discussion see text and Gastaldello et al. 2010). Given the didactic nature of this simulation, background components have not been included; these contributions will make attempts to discriminate between different spectral models even more arduous.

Hereafter we model in a rudimental fashion a multitemperature plasma; our purpose is not to reproduce in detail the thermodynamic structure of the ICM, but to more modestly provide an example of how inhomogeneities characterized by the entropy vs. metallicity anticorrelation can bias abundance measures. We consider a volume of ICM filled with gas at two different densities, the more rarefied component filling most of the volume and the denser filling only 5\%. Since, as already pointed out, sound waves will maintain gas of different densities in approximate pressure equilibrium, overdense region will also be cooler. We assume the hotter component to be at $3.6 \mathrm{keV}$, a relatively low temperature similar to that found in the outer regions of hot clusters, and the cooler to be at half that temperature, i.e. $1.8 \mathrm{keV}$. We also assume the cooler component to have a high metal abundance of $0.4 Z_{\odot}$ and the hotter one to have a lower metallicity of $0.05 Z_{\odot}$, in agreement with the scenario described above. Since the cooler component, filling $5 \%$ of the volume, is twice as dense as the hotter one, its mass will be $10 \%$ of the total mass. We simulated the spectrum from this twotemperature plasma using the fakeit command in the XSPEC spectral fitting package (Arnaud 1996) and using redistribution matrix and effective area files for the EPIC pn. We also verified that, as expected, adopting response files from other CCD instrument does not change our results significantly. By fitting the simulated spectrum with a one-temperature model, we derived a metal abundance in the range $0.2 Z_{\odot}-0.25 Z_{\odot}$. This is a factor of $\sim 3$ larger than the mass weighted metal abundance that can be readily derived from the numbers provided above, i.e. $0.08 Z_{\odot}$ and about a factor of 2 larger than the emission weighted abundance, i.e. $0.11 Z_{\odot}$. The simulated and fit spectra are shown in Fig. 1. As pointed out in Sect. 3.1, spectra from outer regions are typically fit with one-temperature models mostly because 
the statistical quality of the data is insufficient to allow multitemperature fitting. Moreover, even if a multi-temperature fit is attempted, some assumption on the relation between the metallicity of the different components needs to be made as the metal abundance of the different components are largely degenerate with respect to one another. Fitting our simulated spectrum with a two-temperature model, with the two abundances free to vary independently of one another, confirms this. If we assume that the two components have the same abundance we find a metallicity of $0.13 Z_{\odot}$, almost a factor of 2 larger than the input mass weighted abundance.

Despite some disagreement on the steepness of the temperature profiles, (e.g. Walker et al. 2012; Eckert et al. 2013), the shared view is that they decline with radius. This implies that, with the exception of the hottest sytems, the Fe abundance in the outermost regions of many clusters will have to rely on L-shell measures alone. Under such circumstances, constraining the metal abundance is equally if not more arduous. In Eckert et al. (2014) we showed that the spectrum of a particular region could be fit comparably well with a single-temperature model and a multi-temperature one differing by more than a factor of 3 in metal abundance. Before closing this section, it is worth noting that significant biases in the estimate of metal abundances in multi-temperature regions have been recognized and studied for some time (e.g. Buote 2000a,b; Molendi \& Gastaldello 2001; Rasia et al. 2008; Gastaldello et al. 2010). In his seminal papers Buote identified an "Fe-bias" that leads to an underestimation of the Fe abundance, while in Gastaldello et al. (2010) the authors describe an "inverse Fe-bias" that works in the opposite direction, which also happens to be the one at work in our example. What is perhaps less well understood is that biases can be equally important in the outskirts as in the cores of clusters (see Reiprich et al. 2013, for a discussion of this issue).

\subsection{A rough census}

In this section we attempt a first census of metals out to $r_{180}$. As previously discussed, we have good estimates of the metal abundance within $0.2 r_{180}$ from Leccardi et al. (2010). For the region between $0.2 r_{180}$ and $0.4 r_{180}$ we also assume the abundance measured in Leccardi et al. (2010), but we complement it with a factor of 2 uncertainty (see Sect. 3.2). Since we do not have any reliable estimate of the metal abundance beyond $0.4 r_{180}$, we assume it to be anywhere between $0.01 Z_{\odot}$ (i.e. almost no metals ${ }^{6}$ ) and $0.24 Z_{\odot}$ (the value measured between $0.2 r_{180}$ and $\left.0.4 r_{180}\right)$. The upper bound is not based on any data, but on the educated guess that metal abundance profiles do not increase with radius. By combining these estimates with measures of the gas mass within a given radius (Eckert et al. 2012) we derive a mean, cumulative, gas mass weighted, metal abundance profile, $Z_{\mathrm{MW}}(<r)$, out to $r_{180}$ (see Fig. 2). The mean abundance within $r_{180}$, i.e. $Z_{\mathrm{MW}}\left(<r_{180}\right)$, is found to be anywhere between $0.06 Z_{\odot}$ and $0.26 Z_{\odot}$. We point out that, albeit weak, to our knowledge they are the first constraints on the metal content within $r_{180}$.

\section{Discussion}

We have shown that the mean Fe abundance in clusters is very poorly constrained, ranging from a minimum of $0.06 Z_{\odot}$ to a maximum of $0.26 Z_{\odot}$. Although the data used to perform the

\footnotetext{
6 Although the measure on Perseus (Werner et al. 2013) could be significantly biased, the fact that the line has been detected shows that, however little, some Fe must be present.
}

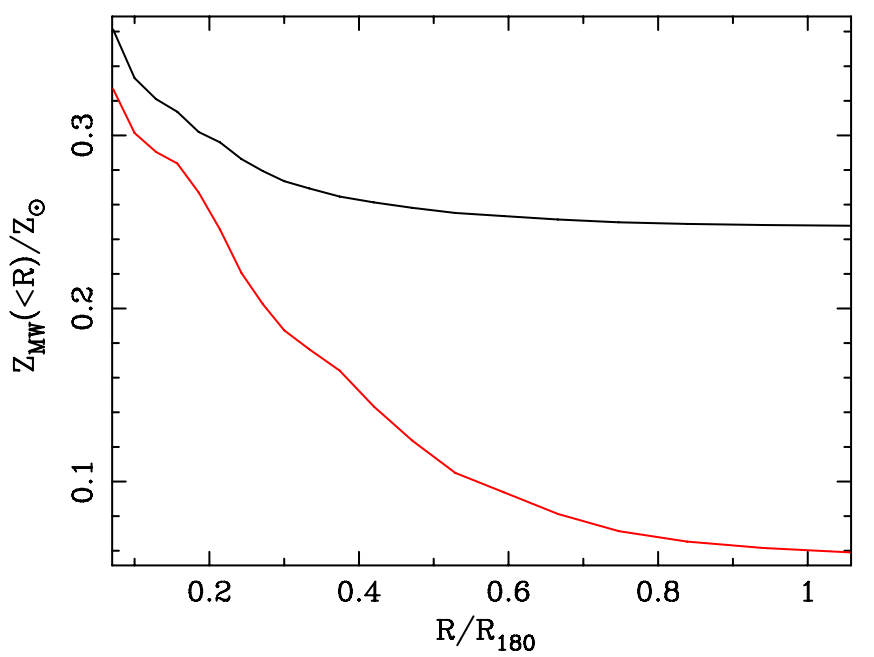

Fig. 2. Mean, cumulative, gas mass weighted, abundance profile for hot clusters. The black and red lines trace, respectively, the rough upper and lower bounds of our estimate.

calculation has been available for some time, and from our own group we might add, this is the first estimate of the mean metal abundance within $r_{180}$. In this section we discuss how, despite their weakness, the limits on the metallicity can provide useful insights.

Over recent decades several authors have discussed the relation between the Fe content of the ICM as estimated from observations, and the value expected from the stellar population observed in clusters (e.g. Renzini 1997; Loewenstein 2013; Renzini \& Andreon 2014). Under the assumption that the most massive systems in our Universe (i.e. rich clusters) are closed boxes, in the sense that material that falls into them can no longer escape, their total $\mathrm{Fe}$ content can be easily predicted if $\mathrm{SNe} \mathrm{Fe}$ yields are known and cluster total stellar masses are measured (e.g. Loewenstein 2013). The authors that have performed this exercise have found that the expected metal content of the ICM falls short of the observed value, assumed to be roughly $0.3 Z_{\odot}$ (e.g. by a factor 2 in Loewenstein 2013). In the previous section we show that while the observed abundance is indeed close to the value adopted by these authors, the mean cluster abundance could differ from it very significantly for the simple reason that the metallicity of the bulk of the ICM has yet to be measured. For example, in a recent paper (Renzini \& Andreon 2014) derive a mean ICM metallicity of $0.3 Z_{\odot}$ by making use of metal abundances measured within $0.6 r_{500}$, i.e. roughly $0.4 r_{180}$ for rich clusters.

If taking an inventory of metals in local clusters is no easy task, trying to establish how abundances vary across cosmic time is even more arduous. There have been several attempts to characterize the redshift evolution of the metallicity (e.g. Balestra et al. 2007; Maughan et al. 2008; Baldi et al. 2012; Ettori et al. 2015). In the latest and most complete of these papers, Ettori et al. (2015), we found no significant evidence of evolution in metallicity in the outermost regions, roughly corresponding to the $0.2 r_{180}-0.4 r_{180}$ range. However, the question of whether and how the bulk of the metal content in clusters, which lies beyond our current reach, varies, remains very much an open one.

It is worth noting that there is other useful information that can be gleaned from the distribution of metals in outskirts. As already discussed in Sect. 1, these measures can be used to gauge the formation and evolution process of cluster in a fashion that is complementary to the one involving the measurement of 
thermodynamic variables. For example, it could be used to test the AGN feedback processes (since metals efficiently trace bulk motions) and pinpoint the metal injection epoch (e.g. Fabjan et al. 2010).

As an interesting aside, we note that in cluster cores the metal budget does not appear to be a problem. Under the assumption that the metal excess observed in cool cores is due to stars currently residing in the BCG invariably found at the centre of these systems (De Grandi et al. 2004), we have found that the measured Fe mass in the ICM is comparable to the expected value (e.g. De Grandi et al. 2014).

\section{Future prospects}

The characterization of metal abundances in cluster outskirts requires data from new experiments that are more sensitive to low surface brightness emission than existing values. The Japanese satellite ASTRO-H (Takahashi et al. 2012) carries several experiments and should be operational within the year. The Soft $\mathrm{X}$-ray Imager (SXI) is comparable to the CCDs on board Suzaku, with one notable exception, the significantly larger field of view $\left(35^{\prime} \times 35^{\prime}\right)$ which may lead to some improvements. The Soft X-ray Spectrometer (SXS) provides unprecedented spectral resolution and will undoubtedly make very significant contributions in several fields (e.g. Kitayama et al. 2014). In the case at hand, however, the relatively high instrumental background combined with the small field of view $\left(3^{\prime} \times 3^{\prime}\right)$ and effective area $\left(300 \mathrm{~cm}^{2}\right.$ at $6 \mathrm{keV}$ ) will make exploration of cluster outer regions with the SXS challenging. Indeed no systematic study of cluster outskirts with SXS is foreseen at this time (Kitayama et al. 2014). The launch of Spektr-RG is currently scheduled for 2017; the eROSITA experiment (Merloni et al. 2012) on board Spektr-RG has characteristics similar to previous CCD imagers and should have a sensitivity to low surface brightness emission comparable to that of the XMM-Newton EPIC cameras.

The ESA mission Athena, currently in its phase A study and with an expected launch in 2028, will carry a Wide Field X-ray Imager (WFI) and an X-ray Integral Field Unit (XIFU). The XIFU is a microcalorimeter array with large collecting area and a $3.5^{\prime} \times 3.5^{\prime}$ field of view. The design of XIFU on board Athena includes an anti-coincidence system and a passive shield, which are expected to reduce the instrumental background by more than an order of magnitude (Lotti et al. 2014). In the current design the WFI enjoys a combination of very large effective area $\left(2 \mathrm{~m}^{2}\right.$ at $\left.1 \mathrm{keV}\right)$ and good angular resolution $\left(\sim 5^{\prime \prime}\right)$ extending over a very large field of view $\left(40^{\prime} \times 40^{\prime}\right)$. Moreover, there are a number of current activities on the telescope and detector design that, if successful, will lead to an experiment with low, stable, and extremely well-characterized background.

While the limited field of view of the XIFU will likely not allow a full coverage of cluster outer regions, its high spectral resolution combined with the low background will permit a sampling of the temperature structure of cluster outskirts. This information can be subsequently fed into the WFI spectral modelling: as pointed out in Sect. 3.2, an adequate characterization of the temperature structure of the plasma is a key point to keep biases in the abundance measurements under control. In Fig. 3 we provide an example of an abundance profile measurements with the WFI on board Athena; details on the properties of the simulated cluster are provided in the figure caption. As shown in the figure, the profile can be measured out to about $r_{180}$. An important point is that the fitting of the WFI spectra was performed with the same spectral model that was used to simulate the data, in the case at hand a one-temperature plasma. Another point worth bearing in

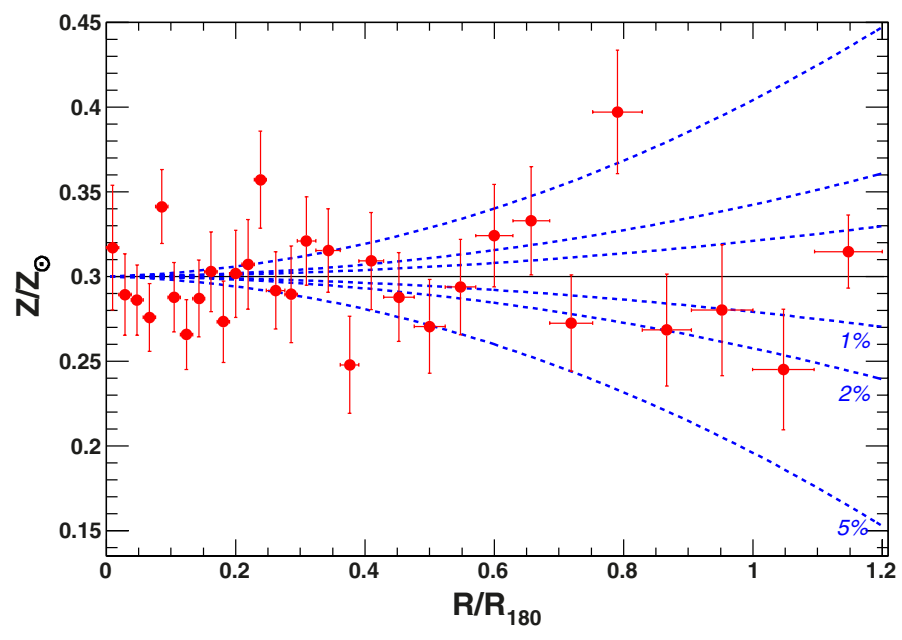

Fig. 3. Fit of a simulated metal abundance profile for an $8 \mathrm{keV}$ cluster at $z=0.1$ for the WFI experiment on Athena. The simulated emissionmeasure and temperature profiles are, respectively, from Eckert et al. (2012) and Reiprich et al. (2013). The spectra were simulated with a one-temperature model and the abundance was fixed to $0.3 Z_{\odot}$ everywhere. The effective area and background intensity are those from the Athena proposal (Rau et al. 2013); the simulated exposure time is $100 \mathrm{ks}$. The data points show the best fitting abundances and related statistical errors assuming perfect reproducibility of the background, the dashed lines refer to the systematic uncertainties associated with different levels of reproducibility of the background, namely $1 \%, 2 \%$, and $5 \%$. At small radii where the source dominates over the background, systematic uncertainties on the latter are of little or no consequence; at large radii, where the background dominates, its reproducibility drives the error budget.

mind is that these measurements depend upon a few critical parameters: a decrease in effective area or, alternatively, an increase in background intensity, will lead to larger errors, that, assuming no change in the level of systematics, cannot be recovered with longer exposure times. Similarly, a loss of reproducibility of the background will lead to an increase in systematic errors; this is illustrated in Fig. 3 where we show that systematic uncertainties grow rapidly in the outskirts as background reproducibility degrades. For instance, at $r_{500}$, which corresponds to $\sim 0.62 r_{180}$, we expect a systematic uncertainty on the measure of the abundance of $2 \%, 4 \%$, and $10 \%$ for a background reproducibility good to $1 \%, 2 \%$, and $5 \%$, respectively, while at $r_{180}$ we expect a systematic uncertainty of $7 \%, 14 \%$, and $35 \%$ for the same levels of background reproducibility. Thus, a de-scoping of the mission entailing a loss in effective area or in background reproducibility, or an increase in background intensity, could significantly limit measures of metal abundances in cluster outskirts.

\section{Summary}

Our results are summarized as follows.

- Robust estimates, characterized by systematic uncertainties of the order of a few percent, are available for cluster core and circum-cores. The mean metal abundance within these regions, i.e. $r<0.2 r_{180}$, is $\sim 0.3 Z_{\odot}$.

- Between 0.2r $r_{180}$ and $0.4 r_{180}$ we have several tens of measurements; however, the limited statistical quality of the data does not allow a detailed reconstruction of the thermodynamic structure of the gas in these regions. This leads to systematic uncertainties in metal abundance measures, which could be as large as a factor of 2 or even more. 
- Since almost nothing is known of the metal abundance beyond $0.4 r_{180}$ where the bulk of the gas mass resides, any claim of disagreement between the measured and expected metal content of the ICM should be viewed with a good dose of skepticism. Using published data and assuming that between $0.4 r_{180}$ and $r_{180}$ the abundance can be constrained between $0.016 Z_{\odot}$ and $0.246 Z_{\odot}$, we have performed a first, albeit rough, census of metals within $r_{180}$. We find the mean, mass weighted abundance within $r_{180}$, i.e. $Z_{\mathrm{MW}}\left(<r_{180}\right)$, to be between $0.06 Z_{\odot}$ and $0.26 Z_{\odot}$; this broad range does not conflict with expectations. Similarly, the question of whether and how the bulk of the metal content in clusters varies with cosmic time has not yet been resolved.

- A solid estimate of abundances in cluster outskirts could be achieved by combining observations of the two experiments that will operate on board Athena, the XIFU and the WFI, provided they do not fall victim to the de-scoping process that has troubled several space observatories over the last decade.

Acknowledgements. S.M. would like to acknowledge the stimulating environment of the workshop "The Metal Enrichment of Diffuse Gas in the Universe" held in Sesto Pusteria in late July 2015, where the original idea for this paper was conceived. We acknowledge financial contribution from contract PRIN INAF 2012 ("A unique dataset to address the most compelling open questions about X-ray galaxy clusters").

\section{References}

Anders, E., \& Grevesse, N. 1989, Geochim. Cosmochim. Acta, 53, 197 Arnaud, K. A. 1996, in Astronomical Data Analysis Software and Systems V, eds. G. H. Jacoby, \& J. Barnes, ASP Conf. Ser., 101, 17 Asplund, M., Grevesse, N., Sauval, A. J., \& Scott, P. 2009, ARA\&A, 47, 481 Baldi, A., Ettori, S., Molendi, S., et al. 2012, A\&A, 537, A142 Balestra, I., Tozzi, P., Ettori, S., et al. 2007, A\&A, 462, 429 Buote, D. A. 2000a, ApJ, 539, 172

Buote, D. A. 2000b, MNRAS, 311, 176

Cora, S. A., Tornatore, L., Tozzi, P., \& Dolag, K. 2008, MNRAS, 386, 96 De Grandi, S., \& Molendi, S. 2001, ApJ, 551, 153

De Grandi, S., \& Molendi, S. 2009, A\&A, 508, 565

De Grandi, S., Ettori, S., Longhetti, M., \& Molendi, S. 2004, A\&A, 419, 7

De Grandi, S., Santos, J. S., Nonino, M., et al. 2014, A\&A, 567, A102 de Plaa, J. 2013, Astron. Nachr., 334, 416

de Plaa, J., Werner, N., Bleeker, J. A. M., et al. 2007, A\&A, 465, 345

Eckert, D., Vazza, F., Ettori, S., et al. 2012, A\&A, 541, A57

Eckert, D., Molendi, S., Vazza, F., Ettori, S., \& Paltani, S. 2013, A\&A, 551, A22

Eckert, D., Molendi, S., Owers, M., et al. 2014, A\&A, 570, A119

Eckert, D., Roncarelli, M., Ettori, S., et al. 2015, MNRAS, 447, 2198

Ettori, S., \& Molendi, S. 2011, Mem. Soc. Astron. It. Supp., 17, 47

Ettori, S., Baldi, A., Balestra, I., et al. 2015, A\&A, 578, A46
Fabjan, D., Borgani, S., Tornatore, L., et al. 2010, MNRAS, 401, 1670 Gaspari, M., Churazov, E., Nagai, D., Lau, E. T., \& Zhuravleva, I. 2014, A\&A, 569, A67

Gastaldello, F., Ettori, S., Balestra, I., et al. 2010, A\&A, 522, A34

Ghizzardi, S., De Grandi, S., \& Molendi, S. 2013, Astron. Nachr., 334, 422

Kaastra, J. 1992, An X-Ray Spectral Code for Optically Thin Plasmas, Internal SRON Leiden Report, updated version 2.0

Kitayama, T., Bautz, M., Markevitch, M., et al. 2014, ArXiv e-prints [arXiv: 1412.1176]

Leccardi, A., \& Molendi, S. 2008a, A\&A, 487, 461

Leccardi, A., \& Molendi, S. 2008b, A\&A, 486, 359

Leccardi, A., Rossetti, M., \& Molendi, S. 2010, A\&A, 510, A82

Liedahl, D. A., Osterheld, A. L., \& Goldstein, W. H. 1995, ApJ, 438, L115

Lodders, K. 2003, ApJ, 591, 1220

Lodders, K. 2010, in Principles and Perspectives in Cosmochemistry, eds. A. Goswami, \& B. E. Reddy, 379

Loewenstein, M. 2013, ApJ, 773, 52

Lotti, S., Macculi, C., Cea, D., et al. 2014, in SPIE Conf. Ser., 9144, 2

Maughan, B. J., Jones, C., Forman, W., \& Van Speybroeck, L. 2008, ApJS, 174, 117

Merloni, A., Predehl, P., Becker, W., et al. 2012, ArXiv e-prints [arXiv: 1209.3114]

Mernier, F., de Plaa, J., Lovisari, L., et al. 2015, A\&A, 575, A37

Mewe, R., Gronenschild, E. H. B. M., \& van den Oord, G. H. J. 1985, A\&AS, 62, 197

Mewe, R., Lemen, J. R., \& van den Oord, G. H. J. 1986, A\&AS, 65, 511

Mitchell, R. J. 1976, Ph.D. Thesis, University College London

Molendi, S. 2004, in Outskirts of Galaxy Clusters: Intense Life in the Suburbs, ed. A. Diaferio, IAU Colloq. 195, 122

Molendi, S., \& Gastaldello, F. 2001, A\&A, 375, L14

Nagai, D., \& Lau, E. T. 2011, ApJ, 731, L10

Planck Collaboration XXIX. 2014, A\&A, 571, A29

Pratt, G. W., Arnaud, M., Piffaretti, R., et al. 2010, A\&A, 511, A85

Rasia, E., Mazzotta, P., Bourdin, H., et al. 2008, ApJ, 674, 728

Rau, A., Meidinger, N., Nandra, K., et al. 2013, ArXiv e-prints [arXiv: 1308.6785$]$

Reiprich, T. H., Basu, K., Ettori, S., et al. 2013, Space Sci. Rev., 177, 1

Renzini, A. 1997, ApJ, 488, 35

Renzini, A., \& Andreon, S. 2014, MNRAS, 444, 3581

Roncarelli, M., Ettori, S., Borgani, S., et al. 2013, MNRAS, 432, 3030

Rossetti, M., \& Molendi, S. 2010, A\&A, 510, A83

Sarazin, C. L. 1988, X-ray emission from clusters of galaxies (Cambridge: Cambridge University Press)

Simionescu, A., Werner, N., Urban, O., et al. 2015, ApJ, 811, L25

Smith, R. K., Brickhouse, N. S., Liedahl, D. A., \& Raymond, J. C. 2001, ApJ, 556, L91

Takahashi, T., Mitsuda, K., Kelley, R., et al. 2012, in SPIE Conf. Ser., 8443, 1

Tamura, T., Kaastra, J. S., den Herder, J. W. A., Bleeker, J. A. M., \& Peterson, J. R. 2004, A\&A, 420, 135

Tamura, T., Hayashida, K., Ueda, S., \& Nagai, M. 2011, PASJ, 63, 1009

Tornatore, L., Borgani, S., Dolag, K., \& Matteucci, F. 2007, MNRAS, 382, 1050

Voit, G. M., Kay, S. T., \& Bryan, G. L. 2005, MNRAS, 364, 909

Walker, S. A., Fabian, A. C., Sanders, J. S., \& George, M. R. 2012, MNRAS, 427, L45

Werner, N., Urban, O., Simionescu, A., \& Allen, S. W. 2013, Nature, 502, 656 